\title{
Overexpression of matrix metalloproteinase-8 and -9 in bronchiectatic airways in vivo
}

\author{
L. Zheng*, W.K. Lam*, G.L. Tipoe*, I.H. Shum*, C. Yan*, R. Leung*, J. Sun*, G.C. Ooi", \\ K.W. Tsang*
}

Overexpression of matrix metalloproteinase- 8 and -9 in bronchiectatic airways in vivo. L. Zheng, W.K. Lam, G.L. Tipoe, I.H. Shum, C. Yan, R. Leung, J. Sun, G.C. Ooi, K.W. Tsang. (C) ERS Journals Ltd 2002.

ABSTRACT: The progressive bronchial dilatation in bronchiectasis is likely to be the result of continued airway matrix destruction, although little is known about the role of neutrophil matrix metalloproteinases (MMPs) in this process.

Immunohistochemistry has been used to investigate the expression and cellular localisation of MMP-8 and MMP-9 in bronchiectatic airways in vivo. Endobronchial biopsies were taken from 25 bronchiectatic patients, and from the right lower lobe in $\mathbf{1 4}$ control subjects. MMP-8, MMP-9, neutrophils and macrophages were stained with monoclonal antibodies and quantified as positive cell $\cdot \mathrm{mm}^{-2}$ of the lamina propria by using an image analysis system.

There were significantly higher densities of MMP-8 and MMP-9 positive cells in the lamina propria of bronchiectatic than control airways. In bronchiectatic airways, the densities of MMP-8 and MMP-9 positive cells correlated with each other and with neutrophil density, but not with macrophage density. In control airways, a significant correlation was found between MMP-8 with neutrophil and MMP-9 with macrophage densities.

An overexpression of neutrophil matrix metalloproteinases in bronchiectatic airways could help explain the continuation of airway destruction in bronchiectasis. In view of the clinical availability of matrix metalloproteinase antagonists, the results presented here could have a significant impact on the development of novel therapies of this untreatable disease.

Eur Respir J 2002; 20: 170-176.

\begin{abstract}
*University Depts of Medicine, " Anatomy and Diagnostic Radiology, The University of Hong Kong, Hong Kong Special Administrative Region, China.

Correspondence: K. Tsang, Associate Professor and Honorary Consultant Physician, University Dept of Medicine, Queen Mary Hospital, The University of Hong Kong, Pokfulam Road, Hong Kong SAR.

Fax: 85228725828

E-mail: kwttsang@hku.hk
\end{abstract}

Keywords: Bronchial biopsy

bronchiectasis

matrix metalloproteinase

neutrophils

Received: September 272001

Accepted after revision: February 7 2002

This study was supported by a Research Grant Council of Hong Kong.
Bronchiectasis is a chronic infective and inflammatory airway disease characterised pathologically by permanent abnormal dilation of the bronchi, and clinically by recurrent purulent sputum production. The pathogenesis of bronchiectasis is not fully understood but destruction of the bronchial wall must be a major contributor to the development of airway dilatation. Neutrophils, the most predominant cells found in the airway lumen of bronchiectasis, possess lytic enzymes, which are likely to play a significant role in the continuous airway damage in bronchiectasis [1-4].

Neutrophil elastase, a neutral serine proteinase, is capable of causing direct lung damage by its lytic actions on connective-tissue proteins of the airway matrix [5]. Several studies have shown overexpression and increased activity of neutrophil elastase in bronchial secretions of patients with bronchiectasis $[1-4,6]$. The authors have also recently shown that sputum elastase levels correlate with those of pro-inflammatory cytokines and disease activity markers, such as $24 \mathrm{~h}$ sputum volume, in steady state bronchiectasis [1]. Neutrophils could also release abundant matrix metallproteinases (MMPs) upon activation [7-10], but little is known of the role of these in the pathogenesis of airway damage in bronchiectasis.

MMPs belong to a family of zinc- and calciumdependent endopeptidases, which are capable of degrading connective tissue [7-10]. MMP-8 (collagenase-2) and MMP-9 (gelatinase B) are produced by neutrophils [7-10], although MMP-8 can be also produced by nonneutrophil-lineage cells such as chondrocytes [11], synovial fibroblasts, and endothelial [12], plasma [13] and airway epithelial cells [14]. In addition, MMP-9 is also expressed by macrophages [7-10]. MMP-8 and MMP-9 are normally stored in the intracellular secondary and tertiary granules respectively, prior to their release by neutrophils [7-10]. MMPs appear to degrade extracellular matrix of articular cartilage, atherosclerotic plaque and tissue surrounding invasive tumours leading to progressive joint destruction in osteoarthritis, rupture of atherosclerotic plague and tumour infiltration of tissue, respectively [9]. Among the collagenases, MMP-8 is the most potent in hydrolysing native type I and II collagens [7, 8]. MMPs also play an important role in the pathogenesis of several respiratory diseases including asthma [15], chronic obstructive pulmonary disease (COPD) [16, 17], adult respiratory distress 
syndrome (ARDS) [9], and pulmonary fibrosis [18]. The levels of MMP-8 and MMP-9 have recently been reported to be raised in the bronchoalveolar lavage fluid (BALF) of patients with bronchiectasis [19, 20], but little is known about their cellular origin and tissue distribution [14]. Therefore, the authors have performed this immunohistochemical study to evaluate the expression and cellular localisation of MMP-8 and MMP-9 in human bronchiectatic airways in vivo.

\section{Methods}

\section{Subject recruitment}

Patients with proven bronchiectasis, diagnosed by high resolution computed tomography (HRCT), were recruited from the respiratory clinics of the University of Hong Kong with written informed consent. Inclusion criteria included: absence of asthma or other unstable systemic diseases, no alteration in medication and dosage for at least 3 months and "steady state" bronchiectasis. The latter was defined as the absence of significant (i.e. $<20 \%$ ) alteration of $24 \mathrm{~h}$ sputum volume, forced expiratory volume in one second (FEV1) and forced vital capacity (FVC), or changes in respiratory symptoms for 3 consecutive weeks [1]. Spirometry (FEV1 and FVC), expressed as \% predicted, was measured between 10:00-12:00 with a SensorMedics 2200 (SensorMedics, Yorba Linda, USA) package. Thoracic HRCT had been performed, within the previous 12 months, using a General Electric Hispeed Advantage Scanner (General Electric, Milwaukee, WI, USA) to perform $1 \mathrm{~mm}$ thick sections at $10 \mathrm{~mm}$ intervals in the supine position. The number of lung lobes (including lingula) affected by bronchiectasis, as evident by the bronchial segment or subsegment being larger than the accompanying artery, was determined for each patient [21].

Subjects, who were otherwise healthy and free from known respiratory diseases, that underwent bronchoscopy for investigation of incidental radiographic discovery of abnormal unilateral shadow suspicious of bronchial carcinoma, were recruited with written informed consent. Other inclusion criteria included the presence of completely normal bronchoscopy and normal lung spirometry. The institutional ethics committee approved the study protocol.

\section{Bronchoscopy and endobronchial biopsy}

All subjects underwent a standard bronchoscopy protocol, without premedications, using Olympus BFP20 bronchoscopes (Olympus, Tokyo, Japan). All endobronchial biopsies (EBBs) were taken with Olympus FB15 alligator forceps (Olympus). For each patient, two to six EBBs were taken from the most severely affected bronchiectatic segments, as determined by HRCT performed $<12$ months before bronchoscopy. Up to four EBBs were obtained from the right lower lobes of basal segments of the radiologically normal bronchial tree from control subjects.

\section{Immunohistochemistry}

EBBs were immediately fixed in $10 \%$ buffered formalin for $2 \mathrm{~h}$ and embedded in paraffin. Immunohistological staining for MMP-8 and MMP-9 was performed using monoclonal mouse antihuman antibodies against MMP-8 (Fuji Chemical Industries Ltd, Japan) and MMP-9 (R \& D System Inc, USA), and amplified using an avidin-biotin peroxidase complex (ABC) method. Isotype control immunoglobulins (Ig) (IgG; Dako, Glostrup, Denmark) were used as negative controls. Serial consecutive $3 \mu \mathrm{m}$ sections were deparaffinised in xylene, rehydrated through graded ethanol solutions, washed with phosphatebuffered saline (PBS), and treated with $0.12 \%$ hydrogen peroxide for $20 \mathrm{~min}$ to block endogenous peroxidase activity. This was followed by preincubation with $20 \%$ normal horse serum for $60 \mathrm{~min}$ to prevent nonspecific binding of immunoglobulins. The sections were then incubated with primary antibodies (diluted 1:400 for MMP-8 and 1:40 for MMP-9 in PBS) at $4{ }^{\circ} \mathrm{C}$ in a moist chamber over night, and washed again with PBS. This was followed by incubation with biotinylated "Universal" antibody and with Vectastain Elite $\mathrm{ABC}$ reagent (VectaStain Universal Elite ABC kit; Vector Laboratories Inc, Burlingame, CA, USA) respectively for $45 \mathrm{~min}$. Diaminobenzidine (Vector Laboratories Inc) was used as substrate. Slides were counterstained with haematoxylin and mounted in synthetic mounting medium.

Consecutive sections were also stained for neutrophils and macrophages with murine monoclonal antibodies against human neutrophil elastase (1:600 in PBS) and macrophages (CD68, 1:100 in PBS) (Dako) as outlined above, to investigate the inflammatory infiltrate in bronchiectasis and cellular localisation of MMP-8 and MMP-9.

\section{Quantitation of immunostaining}

Positive staining for MMP-8, MMP-9, neutrophils and macrophages was scored if the staining was exclusively intracytoplasmic [22]. To minimise variation in interpretation of the slides, staining for each marker from both patients and controls was done in one single experiment. Slides were coded and then assessed by a single observer, who was blind to the origin of the bronchial biopsies. The total number of cells that stained positive for MMP-8, MMP-9, neutrophil-elastase and CD68 in the lamina propria of EBB sections were counted by using a Leica Qwin computerised image analyser (Image Analysis, Cambridge, UK) at a final magnification of $\times 400$. Results were expressed as positive cell density and calculated as positive cells $\cdot \mathrm{mm}^{-2}$ of lamina propria.

\section{Statistical analysis}

Results were expressed as median and range unless otherwise specified. Comparison between groups was made nonparametrically by using the Mann-Whitney 

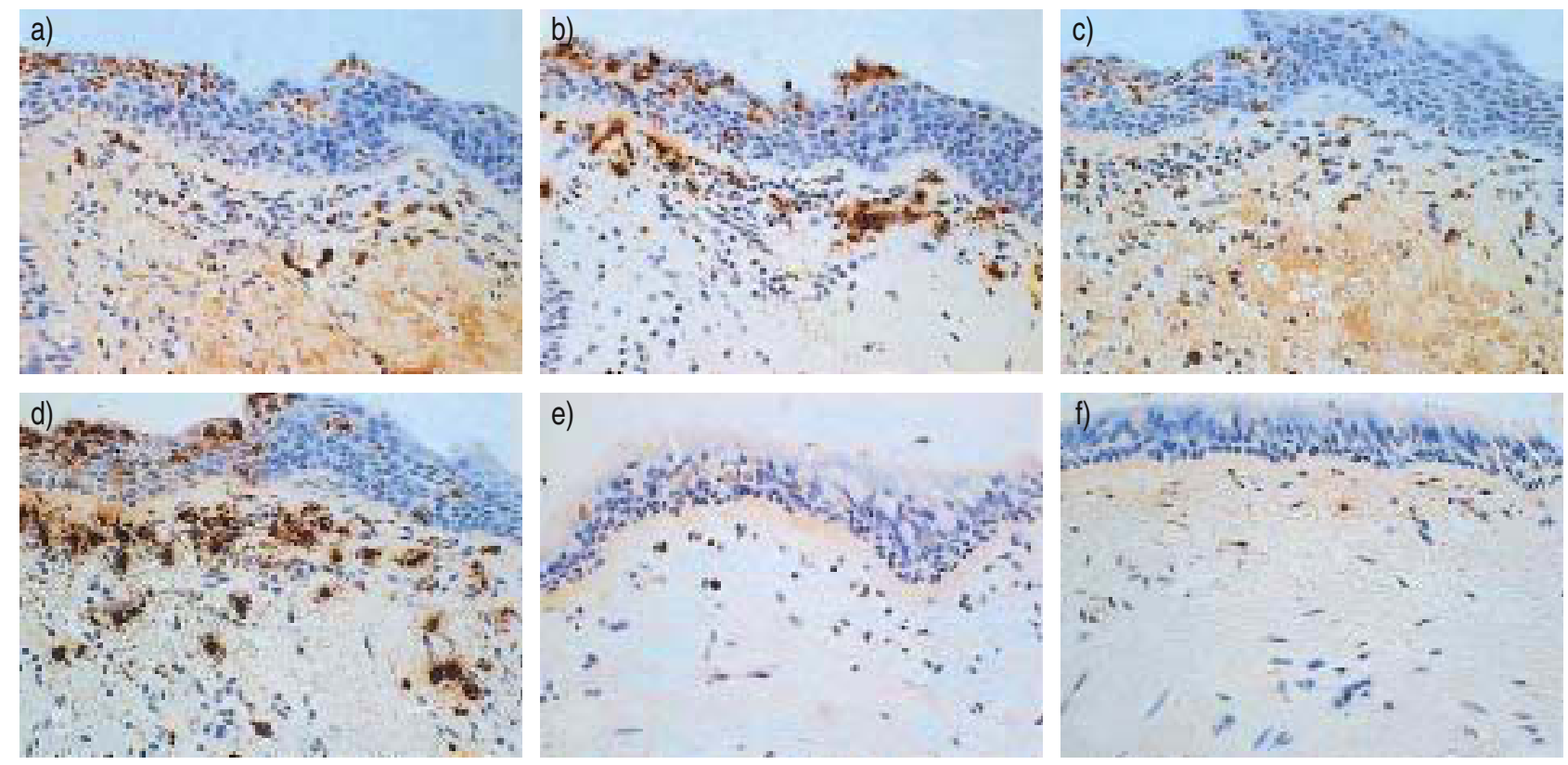

Fig. 1.-Photomicrographs of immunostaining on bronchial biopsy specimens obtained from the same bronchiectatic subject showing a) matrix metalloproteinase (MMP)-8 protein and b) neutrophil elastase expression in sequential sections, and c) MMP-9 protein and d) neutrophil elastase expression in sequential sections. These sections reveal co-localisation of MMP-8 and MMP-9 with neutrophil stain. Figures e) and f) show immunostaining for MMP-8 and MMP-9 proteins in control bronchial biopsies. Each original magnification $\times 400$.

rank-order test. Correlation was evaluated by Spearman's rank method. A p-value of $<0.05$ was considered as statistically significant.

\section{Results}

\section{Patient demography and clinical characteristics}

Twenty-five patients with bronchiectasis (13 females, mean \pm SD age $64 \pm 12$ yrs, FEV1 $79.3 \pm 27.6 \%$, FVC $82 \pm 25.8 \%$ ) and 14 control subjects (five females, mean \pm SD age $58 \pm 12$ yrs) were recruited between September 1998 and February 2000. Seventeen of the patients were nonsmokers, five were exsmokers for at least 3 yrs, and three were current smokers. There was no significant difference in age $(p=0.11)$, sex distribution $(\mathrm{p}=0.33)$ or smoking history $(\mathrm{p}=$ 0.78 ) between the two subject groups. Most patients had bilateral bronchiectasis, and the biopsied bronchial segments were right lower $(n=16)$, left lower $(n=2)$, lingula $(n=2)$, right middle $(n=4)$ and right upper $(n=1)$ lobes. Among the bronchiectatic subjects, 11 were on no regular medications while the others were on regular inhaled bronchodilator $(n=4)$, oral theophylline $(n=3)$ and diuretic therapy $(n=2)$.

\section{Expression and localisation of metalloproteinase- 8 and -9 in bronchiectatic airways}

Figure 1 shows the expression and localisation of MMP-8 and MMP-9 in both the control and bronchiectatic airway biopsies. There were significantly higher densities of MMP-8 and MMP-9 positive cells in the lamina propria of bronchiectatic (median 275, range 39-816 cells $\cdot \mathrm{mm}^{-2}$ and $291,68-837$ cells $\cdot \mathrm{mm}^{-2}$, respectively) than in control airways (66, $12-163 \mathrm{cells} \cdot \mathrm{mm}^{-2}$ and $67,23-202$ cells $\cdot \mathrm{mm}^{-2} ; \mathrm{p}<0.001$ for both; fig. 1a, c, e and f; table 1). In the bronchiectatic airways, cells that stained positive for MMP-8 and MMP-9 were found predominantly at the subepithelial areas, similar to neutrophils, and some were also found within the epithelial layer (fig. 1a-d). Sequential sections stained for neutrophil elastase showed that positive staining for MMP-8 and MMP-9 co-localised with that of neutrophil elastase in bronchiectatic airways (fig. 1a-d). Intravascular neutrophils that stained positive for MMP-8 and MMP-9 were also detected in bronchiectatic airways.

\section{Neutrophil and macrophage infiltration in bronchiectatic airways}

Significantly higher densities of neutrophils and macrophages were found in the lamina propria

Table 1.-Results of immunostaining in bronchial mucosa of subjects who underwent bronchial biopsy

\begin{tabular}{lccc}
\hline $\begin{array}{l}\text { Positive } \\
\text { cell·mm } \\
\text { of lamina } \\
\text { propria }\end{array}$ & $\begin{array}{c}\text { Bronchiectasis } \\
\text { patients }\end{array}$ & Controls & p-values \\
\hline Subjects n & 25 & & \\
MMP-8 & $275(39-816)$ & $66(12-163)$ & 0.0001 \\
MMP-9 & $291(68-837)$ & $67(23-202)$ & 0.0000 \\
Neutrophils & $539(101-1493)$ & $115(24-316)$ & 0.0000 \\
Macrophages & $736(145-1602)$ & $339(129-856)$ & 0.0003 \\
\hline
\end{tabular}

Data are presented as median (range). MMP: matrix metalloproteinase. 
Table 2. - Summary of correlation analysis for parameters assessed in bronchiectasis and control subjects

\begin{tabular}{|c|c|c|}
\hline & $\begin{array}{c}\text { MMP-8 } \\
\mathrm{r} \text { (p-value) }\end{array}$ & $\begin{array}{c}\text { MMP-9 positive cells } \cdot \mathrm{mm}^{-2} \\
\mathrm{r}(\mathrm{p} \text {-value })\end{array}$ \\
\hline \multicolumn{3}{|l|}{ Bronchiectasis } \\
\hline MMP-8 positive cells $\cdot \mathrm{mm}^{-2}$ & & $0.74(<0.001)^{\#}$ \\
\hline MMP-9 positive cells $\cdot \mathrm{mm}^{-2}$ & $0.74(<0.001)^{\#}$ & \\
\hline Neutrophils $\cdot \mathrm{mm}^{-2}$ & $0.69(<0.001)^{\#}$ & $0.66(<0.001)^{\#}$ \\
\hline Macrophages $\cdot \mathrm{mm}^{-2}$ & $0.39(0.06)$ & $0.25(0.22)$ \\
\hline FEV1 $\%$ pred & $-0.07(0.77)$ & $-0.12(0.60)$ \\
\hline FVC $\%$ pred & $-0.13(0.55)$ & $-0.18(0.41)$ \\
\hline Lung lobes with bronchiectasis $n$ & $-0.11(0.62)$ & $0.05(0.82)$ \\
\hline \multicolumn{3}{|l|}{ Controls } \\
\hline MMP- 8 positive cells $\cdot \mathrm{mm}^{-2}$ & & $0.35(0.22)$ \\
\hline MMP-9 positive cells $\cdot \mathrm{mm}^{-2}$ & $0.35(0.22)$ & \\
\hline Neutrophils $\cdot \mathrm{mm}^{-2}$ & $0.75(0.002)^{\#}$ & $-0.015(0.96)$ \\
\hline Macrophages $\cdot \mathrm{mm}^{-2}$ & $0.39(0.17)$ & $0.63(0.02)^{\#}$ \\
\hline
\end{tabular}

Data are presented as Spearman's rank correlation coefficients. MMP: matrix metalloproteinase; FEV1: forced expiratory volume in one second; FVC: forced vital capacity. ${ }^{\#}: \mathrm{p}<0.05$.

of bronchiectatic, when compared with control, airways ( $<<0.001$ for both; table 1$)$. Unlike neutrophils, macrophages appeared to be evenly distributed throughout the entire bronchial lamina propria in both bronchiectatic and control airways.

\section{Correlation analysis}

Table 2 summaries the results of correlation analysis of the study. In the bronchiectatic airways, there was a significant correlation between the densities of MMP-8 and MMP-9 positive cells (fig. 2a). In addition, both MMP-8 and MMP-9 positive cell densities independently correlated with neutrophil density (fig. 2b and c). In control airways, there was no correlation between MMP-8 and MMP-9 positive cell densities (table 2). However, MMP-8 positive cell density correlated with neutrophil density, and MMP-9 with CD68 positive cell density (fig. 3a and $\mathrm{b}$ ). There was no correlation between MMP-8 and MMP-9 positive cell densities with macrophage density, or correlation between FEV1, FVC and number of lobes affected by bronchiectasis with MMP-8 and MMP-9 positive cell densities among the bronchiectasis patients (table 2).

\section{Discussion}

The data from this study showed an upregulation of MMP-8 and MMP-9 expression in the bronchiectatic compared with control airways. The increased expression of MMP-8 and MMP-9 in the bronchiectatic airways correlated significantly with increased airway neutrophils, but not with airway macrophages, although airway macrophage density was also markedly increased in bronchiectasis. Sequential sections stained for neutrophil elastase, MMP-8 and MMP-9 showed that positive staining co-localised, therefore suggesting that MMP-8 and MMP-9 containing cells were neutrophils in bronchiectasis. However, there was no correlation between the airway expressions of MMP-8 and MMP-9 with FEV1\% pred, FVC\% pred and the number of bronchiectatic lung lobes in bronchiectasis.

Despite considerable recent progress made in the understanding of airway inflammation in asthma and COPD, little is known of this in bronchiectasis. Most previous studies on airway inflammation in bronchiectasis have focused on measuring cytokine profiles in sputum and blood $[1-5,6,23]$, rather than studying airway wall inflammations. The results in this study support previous reports [24-26] as neutrophils are not only predominant in the airway lumen of bronchiectatic patients, but they are also abundant in the airways. Macrophage density was also significantly increased in the bronchiectatic airway, which strongly suggests an important pathogenic role for macrophages in bronchiectasis.

Structural changes or remodelling of the bronchi occur as a result of continued tissue destruction and repair processes. Permanent and pathological dilatation of bronchi is unique in bronchiectasis, and only occurs if destruction of connective tissues overwhelms the repair process. Lytic enzymes released by activated airway neutrophils, that are capable of digesting the airway wall matrix, are likely to contribute to this process. In COPD, which is increasingly being recognised to display intense neutrophil airway infiltration [27], there is increased expression of MMP-8 and MMP-9 in sputum, BALF and airway biopsies $[16,17]$. The excessive release of neutrophilic lytic enzymes has also been strongly implicated in the development of emphysema [27, 28]. The results here showed, for the first time on airway biopsies, that expression of neutrophil MMPs is upregulated in bronchiectatic airways, which could be the missing link between intense neutrophil infiltration and destruction of airways in the pathogenesis of bronchiectasis.

Although the levels of MMP-8 and MMP-9 in BALF of patients with bronchiectasis have been evaluated, [14, 19, 20], little was known about the cellular localisation and expression of these MMPs in the bronchiectatic airways until PRIKK et al. [14] reported their findings on resected bronchiectatic 
a)

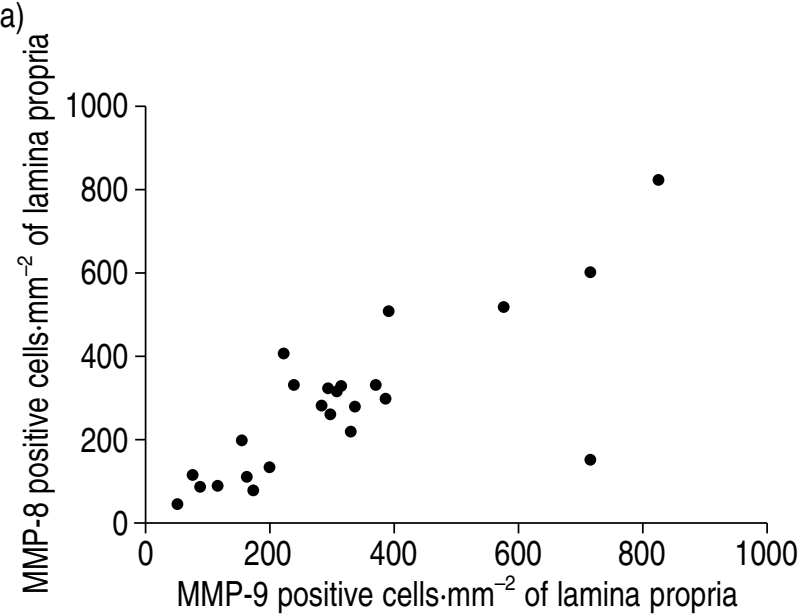

b)

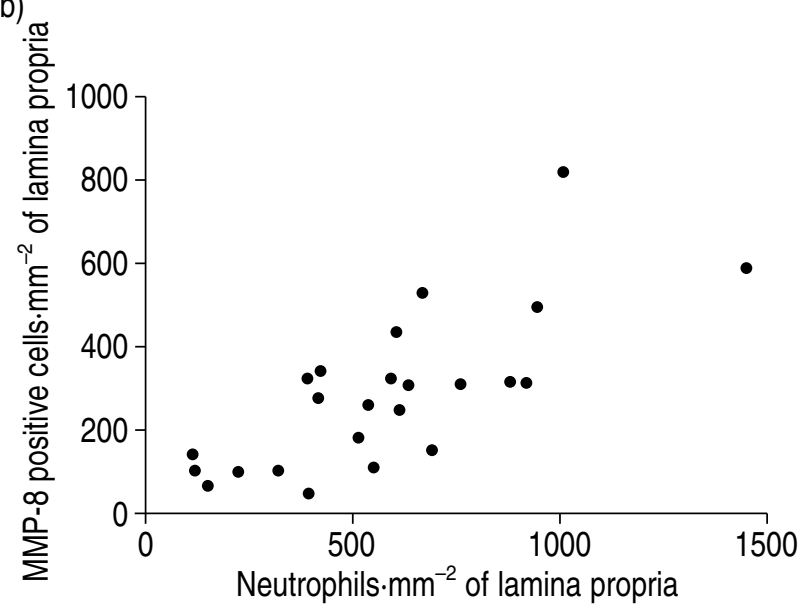

c)

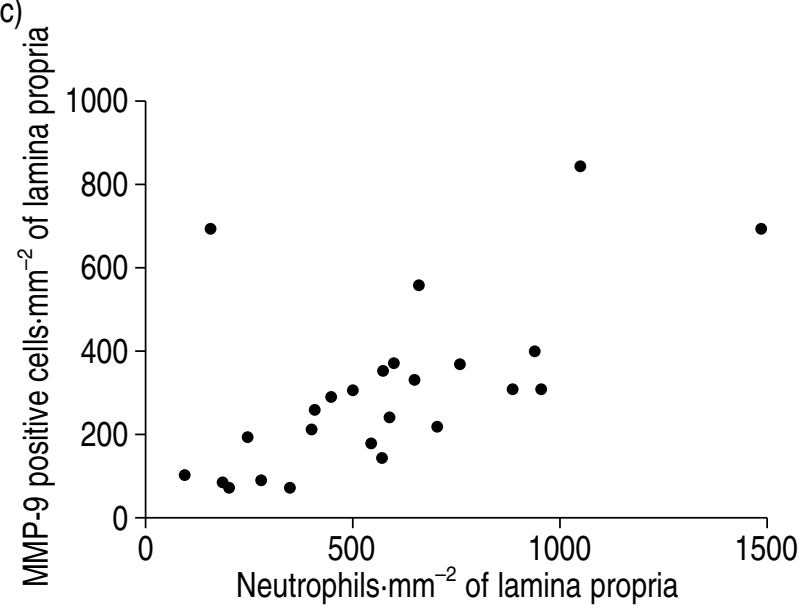

Fig. 2.-Scatterplots showing the correlation between a) matrix metalloproteinase (MMP)-8 and MMP-9 positive cells $(r=0.74$, $\mathrm{p}<0.001)$, b) MMP-8 positive cells and neutrophils $(\mathrm{r}=0.69$, $\mathrm{p}<0.001$ ), and c) MMP-9 positive cells and neutrophils in bronchial biopsy specimens obtained from bronchiectatic subjects $(\mathrm{r}=0.66, \mathrm{p}<0.001)$.

lungs. The data in this study, obtained from evaluation of control airways showed that MMP-8 positive cells correlated with neutrophil densities and MMP-9 positive cells with macrophage densities. This suggests

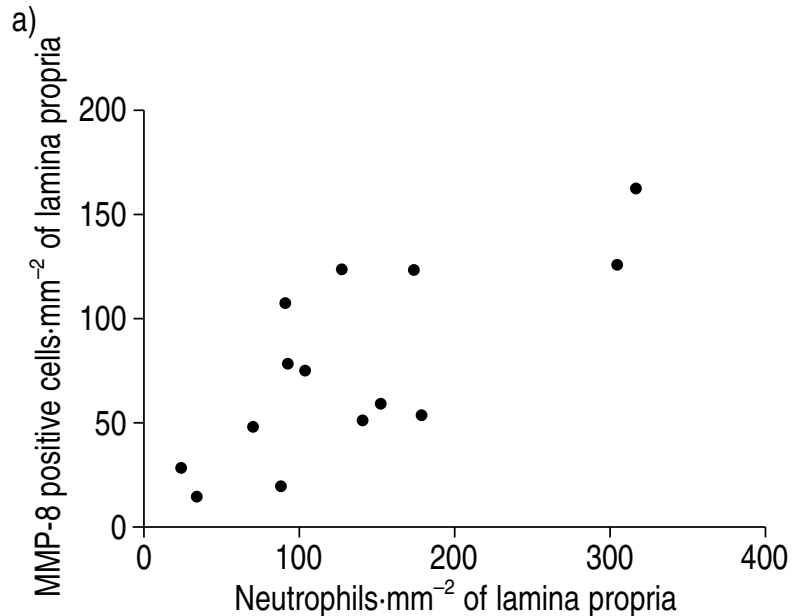

b)

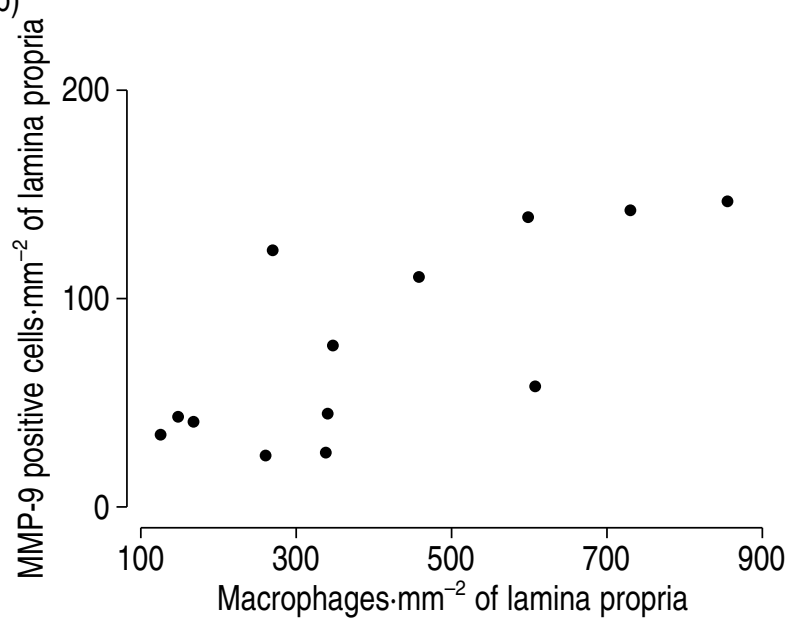

Fig. 3.-Scatterplots showing the correlation between a) matrix metalloproteinase (MMP)- 8 positive cells and neutrophils ( $r=0.75$, $\mathrm{p}=0.002$ ), and b) MMP-9 positive cells and macrophages in bronchial biopsy specimens from control subjects $(r=0.63, p=0.02)$.

that MMP-9 could be predominantly produced by airway macrophages, while MMP-8 could be solely produced by neutrophils in normal airways. In the bronchiectatic airways, the correlation studies showed another pattern. There was a correlation between MMP-8 and MMP-9 expression with neutrophil density, and also co-localisation of these MMPs within neutrophils. A recent study employing immunohistochemistry and in situ hybridisation showed that MMP-8 protein and messenger ribonucleic acid (mRNA) expression occurred not only in neutrophils and macrophages, but also in bronchial epithelium obtained from resected bronchiectatic tissue [14]. However, the latter finding was not confirmed by the data in this study, as little if any expression of these MMPs in bronchial epithelium was found (fig. 1a-d ). This could be explained by the fact that a highly specific monoclonal antihuman neutrophil MMP-8 was used, rather than a polyclonal antibody, which was employed by PRIKK et al. [14] in their immunohistochemistry protocol. While five and two patients were evaluated for MMP-8 protein and mRNA expression, respectively by PRIKK et al. 
[14], bronchiectatic tissue obtained from 25 patients was assessed here. The patients in this study were also stringently judged to be in stable condition prior to undertaking bronchial biopsies, but it is unclear if the five patients in the PRIKK et al. [14] series were stable prior to their resection, and whether or not they were on antibiotics or anti-inflammatory treatment, which could potentially affect MMP expression. The data here strongly suggest that neutrophils, rather than airway epithelium, are most likely to be the major source of MMP-8 and MMP-9 in the bronchiectatic airways.

Neutrophil MMPs are stored in intracellular granules and released upon stimulation by factors affecting neutrophil activation such as tumour necrosis factor (TNF)- $\alpha$ and interleukin (IL)-1 $\beta$ [7-10], which are abundant in the bronchiectatic airways $[1,29]$. As neutrophil MMPs have to be activated in the extracellular environment before they become catalytic [7-10], the intracellular MMP-8 and MMP-9 showed in this study are most likely to be in inactive forms, contrary to the activated form of MMP-8 demonstrated by western immunoblotting technique in BALF obtained from patients with bronchiectasis $[14,19]$. As there is no published data on the activity of intracellular MMPs in bronchiectatic airways, most probably due to the poor availability of bronchial biopsies, it would be important to evaluate this in the future. As the control subjects in this study did not produce any sputum, the levels of activated MMP-8 and MMP-9 in sputum, i.e. extracellular MMPs, between control and bronchiectasis subjects could not be evaluated. However, plasmin, plasma kallikrein, cathepsin B, cathepsin $G$ and neutrophil elastase, which are also abundant in the bronchiectatic airways $[1-4,6,30]$, have been implicated in the activation of neutrophil MMPs in vivo [7, 8]. It is, therefore, very likely that the abundant airway levels of these would serve to act as activators of MMP-8 and MMP-9 upon their release from neutrophils in the bronchiectatic airways.

Although patients with more severely affected bronchiectasis, defined as those with more sputum production and more frequent exacerbations, have been reported to have more activated MMP-8 in BALF than their counterparts [19], the results of this study show no relationship between the airway neutrophil MMP-8 and MMP-9 expression with FEV1\%, FVC\% and number of affected lung lobes among the bronchiectatic patients. This lack of correlation could be due to the fact that these relatively crude clinical parameters merely reflect the end results of the bronchiectatic process, rather than the continued airway damage. In addition, BALF MMPs could be derived from a number of sources such as alveolar cells and luminal leukocytes, which are exposed to the contents of bronchial lumen including bacteria and their products and pro-inflammatory mediators. BALF MMP levels, therefore, do not necessarily reflect those within the wall of the bronchial mucosa. A lack of correlation between sputum MMP-9 concentration and FEV1 has also been reported among patients with asthma and chronic bronchitis [17].

The original data here shows an upregulation of intraneutrophilic metalloproteinase- 8 and -9 in bronchiectatic airways. These changes could explain the gradual airway destruction in bronchiectasis. Further studies are needed to investigate the possible imbalance between neutrophil metalloproteinases and their natural inhibitors (tissue inhibitors of metalloproteinases) in the development of bronchiectasis. As metalloproteinase inhibitors are now available for clinical trials in the treatment of rheumatoid arthritis and osteoarthritis [9], it is possible that they can also be a novel therapy in bronchiectasis.

\section{References}

1. Tsang KW, Chan K, Ho P, et al. Sputum elastase in steady-state bronchiectasis. Chest 2000; 117: 420-426.

2. Stockley RA, Hill SL, Morrison HM, Starkie CM. Elastolytic activity of sputum and its relation to purulence and to lung function in patients with bronchiectasis. Thorax 1984; 39: 408-413.

3. Lloberes P, Montserrat E, Montserrat JM, Picado C. Sputum sol phase proteins and elastase activity in patients with clinically stable bronchiectasis. Thorax 1992; 47: 88-92.

4. Stockley RA. The role of proteinases in the pathogenesis of chronic bronchitis. Am J Respir Crit Care Med 1994; 150: S109-S113.

5. Brown GM, Donaldson K. Degradation of connective tissue components by lung derived leucocytes in vitro: role of proteases and oxidants. Thorax 1988; 43: 132139.

6. Sepper R, Konttinen YT, Ingman T, Sorsa T. Presence, activities, and molecular forms of cathepsin G, elastase, alpha 1-antitrypsin, and alpha 1-antichymotrypsin in bronchiectasis. J Clin Immunol 1995; 15: 27-34.

7. O'Connor CM, FitzGerald MX. Matrix metalloproteases and lung disease. Thorax 1994; 49: 602609.

8. Birkedal-Hansen H. Proteolytic remodeling of extracellular matrix. Curr Opin Cell Biol 1995; 7: 728-735.

9. Shapiro SD, Senior RM. Matrix metalloproteinases. Matrix degradation and more. Am J Respir Cell Mol Biol 1999; 20: 1100-1102.

10. Corbel M, Lagente V, Boichot E. Pulmonary inflammation and tissue remodelling: role of metalloproteinases. Eur Respir Rev 2000; 10: 260-263.

11. Chubinskaja S, Huch K, Mikecz K, et al. Chrondrocyte matrix metalloproteinase-8. Upregulation of neutrophil collagenase by interleukin-1 beta in human cartilage from knee and ankle joints. $L a b$ Invest 1996; 74: 232-240.

12. Hanemaaijer $\mathrm{R}$, Soorsa $\mathrm{T}$, Konttinen $\mathrm{YT}$, et al. Matrix Metalloproteinase- 8 is expressed in rheumatoid synovial fibroblasts and endothelial cells. $\mathrm{J} \mathrm{Bio}$ Chemistry 1997; 272: 31504-31509.

13. Wahlgern J, Maisi P, Sorsa $\mathrm{T}$, et al. Expression and induction of collagenases (MMP-8 and -13) in plasma cells associated with bone-destructive lesions. J Pathology 2001; 194: 217-224.

14. Prikk K, Maisi P, Pirila E, et al. In vivo collagenase-2 (MMP-8) expression by human bronchial epithelial cells and monocytes/macrophages in bronchiectasis. J Pathology 2001; 194: 232-238.

15. Hoshino M, Nakamura Y, Sim J, Shimojo J, Isogai S. Bronchial subepithelial fibrosis and expression of 
matrix metalloproteinase-9 in asthmatic airway inflammation. J Allergy Clin Immunol 1998; 102: 783-788.

16. Segura-Valdez L, Pardo A, Gaxiola M, Uhal BD, Bercerril C, Selman M. Upregulation of gelatinases A and $\mathrm{B}$, collagenases 1 and 2, and increased parenchymal cell death in COPD. Chest 2000; 117: 684-694.

17. Vignola AM, Riccobono L, Mirabella A, et al. Sputum metalloproteinase-9/tissue inhibitor of metalloproteinase-1 ratio correlates with airflow obstruction in asthma and chronic bronchitis. Am J Respir Crit Care Med 1998; 158: 1945-1950.

18. Lemjabbar H, Gosset $\mathrm{P}$, Lechapt-Zalcman E, et al. Overexpression of alveolar macrophage gelatinase B (MMP-9) in patients with idiopathic pulmonary fibrosis: effects of steroid and immunosuppressive treatment. Am J Respir Cell Mol Biol 1999; 20: 903-913.

19. Sepper R, Konttinen YT, Ding Y, Takagim M, Sorsa T. Human neutrophil collagenase (MMP-8), identified in bronchiectasis BAL fluid, correlates with severity of disease. Chest 1995; 107: 1641-1647.

20. Sepper R, Konttinen YT, Sorsa T, Koski H. Gelatinolytic and type IV collagenolytic activity in bronchiectasis. Chest 1994; 106: 1129-1133.

21. McGuniness G, Naidich DP, Leitman BS, McCauley DI. Bronchiectasis: CT evaluation. Am J Radiol 1993; 160: 253-259.

22. Zheng L, Walters EH, Ward C, et al. Airway neutrophilia is present in stable and Bronchiolitis Obliterans Syndrome patients post-lung transplant. Thorax 2000; 55: 53-59.
23. Ho PL, Chan KN, Ip MS, et al. The effect of Pseudomonas aeruginosa infection on clinical parameters in steady-state bronchiectasis. Chest 1998; 114 : 1594-1598.

24. Gaga M, Bentley AM, Humbert M, et al. Increases in CD4+ T lymphocytes, macrophages, neutrophils and interleukin 8 positive cells in the airways of patients with bronchiectasis. Thorax 1998; 53: 685-691.

25. Eller J, Lapa-e-Silva JR, Poulter LW, Lode H, Cole PJ. Cells and cytokines in chronic bronchial infection. Ann NY Acad Sci 1994; 725: 331-345.

26. Lapa de Silva JR, Jones JAH, Cole PJ, Poulter LW. The immunological component of cellular inflammatory infiltrate in bronchiectasis. Thorax 1989; 44: 668-673.

27. Stockley RA. Neutrophils and protease/antiprotease imbalance. Am J Respir Crit Care Med 1999; 160: S49_ S52.

28. Nadel JA. Role of neutrophil elastase in hypersecretion during COPD exacerbations, and proposed therapies. Chest 2000; 117: 386S-389S.

29. Tsang KW, Ho PL, Lam WK, et al. Inhaled fluticasone reduces sputum inflammatory indices in severe bronchiectasis. Am Respir Crit Care Med 1998; 158: 723-727.

30. Sepper R, Konttinen YT, Buo L, et al. Potentiative effects of neutral proteinases in an inflamed lung: relationship of neutrophil procollagenase (proMMP-8) to plasmin, cathepsin $\mathrm{G}$ and tryptase in bronchiectasis in vivo. Eur Respir J 1997; 10: 2788-2793. 